B.E. Lautrup, A. Peterman and E. de Rafael CERN - Geneva

The contribution to the snomalous magnetic moment of the muon from the Feynman diagrams shown in the Figure is found to be $\left(\frac{\boldsymbol{\alpha}}{\boldsymbol{\pi}}\right)^{3} \quad\left[-\frac{28}{9}+\frac{4}{9} \boldsymbol{\pi}^{2} \log 2-\frac{2}{3} \boldsymbol{3}(3) \log \left(\frac{\mathrm{m} \boldsymbol{\mu}}{\mathrm{m}_{\mathrm{e}}}\right)+0.68 \pm 0.01\right.$. 
The total sixth-order contribution to the anomalous magnetic moment of the muon, $\frac{1}{2}\left(g_{p}-2\right)$, can be split into two parts

$$
1 / 2\left(g_{\mu}-2\right)=1 / 2\left(g_{e}-2\right)+1 / 2\left(g_{\mu}-g_{e}\right) \text {. }
$$

Here we are concerned with sixth-order contributions to the difference of muon and electron $g$ factors, $\frac{1}{2}\left(g_{\boldsymbol{\mu}}-g_{e}\right) \equiv a_{\mu}-a_{e}$. There are twenty-four Feynman diagrams contributing at that order. They can be classified in three groups :

i) fourth-order vacuum polarization insertions into the secondorder anomalou.s magnetic moment (four diagrams);

ii) light by light scattering contributions (six diagrams);

iii) secoud-order vacuidm polarization insertions into the fourth-order anomalous magnetic moment (fourteen diagrams)。

The contributions from $i$ ) and $i i$ ) have already been calculated 1),2). The total contribution from iii) is known to be of the form 3 )

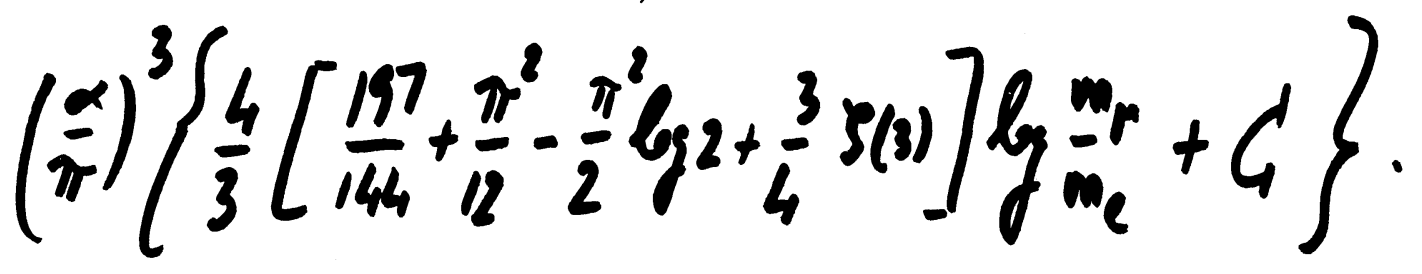

However, the contribution to $C$ is only known for two of the fourteen diagrams 4 )。

The purpose of this note is to present the results of a calcula. tion of the contributions to $C$ from the Feynman diagrams shown in Fig. 1。 We find

$$
C(\text { Fig. })=+0.68 \pm 0.01
$$




\section{DESCRIPTION OF CALCULATIONS}

We have calculated the contribution to the anomalous magnetic moment of the muon, $\frac{1}{2}\left(g_{\mu}-2\right)$, from the Feynman diagrams shown in Fig. 1. The results for each individual diagram are given in the Table. The total contribution from these diagrams, which has no infrared divergences and is gauge invariant, is

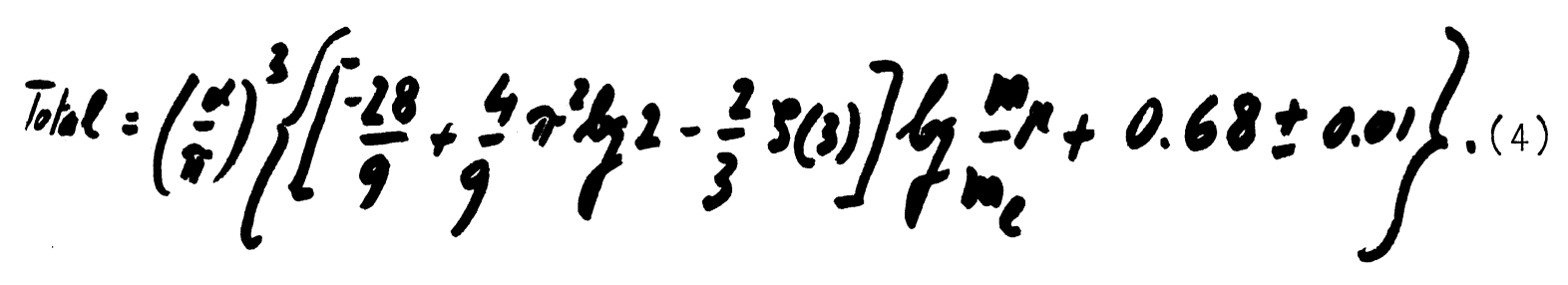

Numerically

$$
\text { Tokl }=(-3.97 \pm 0.01)\left(\frac{\alpha}{\pi}\right)^{3}=(-4.78 \pm 0.01) \mathrm{N}^{-8}
$$

The calculations were done partially analytically and partially numerically. For each diagram at least two different parametrizations were made. The infra-red terms and terms depending on $\log \left(\mathrm{m}_{\boldsymbol{\mu}} / \mathrm{m}_{e}\right)$ were extracted analytically and checked numerically. The constant terms were evaluated numerically, using the ISD integration subroutine 5). In all cases it was possible to reduce these terms to at most fivedimersional integrals.

The reduction of graphs $(a),(b),(e)$ and $(f)$ to parametric form involves little Dirac algebra and it was done by hand ir all cases. One method was to use Feynman rules. Another, was to combine dispersion techniques for the self-energy insertion and vacuum polarization insertion with Feynman parametrization of the vertex involving the external electromagnetic field. The second method leads very quickly to twodimensional integrals which, in principle, can be áone analytically; however, for numerical integration it has the disadvantage that the integrand involves irrational as well as logarithmic functions with strong cancellations between different terms. 
The reduction of graphs (c), (d), (g) and ( $h$ ) to parametric form is more laborious. It was done by hand, and also using the computer program SCHOONSCHIP developed by Veltman 6). Different parametrizationi were obtained by combining the renormalized vertex insertion with the external vertex in various ways.

The contribution to $\frac{1}{2}\left(g_{\mu}-2\right)$ from diagrams $(a),(c),(e)$ and $(g)$ are respectively equal. to those from diagrams $(b),(d),(f)$ and $(h)$. In the Feynman gauge, diagrams $(a),(b),(c)$ and (d) are infra-red divergent; however, the divergences disappear when the self-enerin insertion and the vertex insertion are taken together. Similarly, diagrams $(e),(f),(g)$ and $(\mathrm{h})$ have $\log ^{2}\left(\mathrm{~m} \boldsymbol{\mu} / \mathrm{m}_{e}\right)$ dependences which also disappear when the self-energy insertion and the vertex insertion are taken together.

The coefficient of the $\log \left(\mathrm{m} \mu / \mathrm{m}_{e}\right)$ term in the total contribution given in Eq. (1) is precisely $4 / 3(\boldsymbol{\alpha} / \boldsymbol{R})$ times the fourth-order contribution to the anomalous magnetic moment from the self-energy and vertex insertion graphs. This is in agreement with the results which one obtains applying the techniques of the renormalization group 3 ).

The purpose of this calcrlation was to obtain the remainder term for which we find

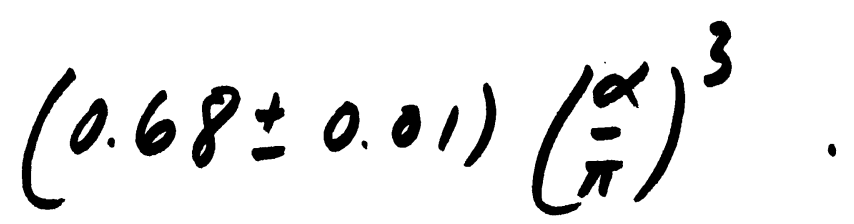

The error is due to the numerical integrations. This term has opposite sign to the $\log \left(\mathrm{m}_{\boldsymbol{\mu}} / \mathrm{m}_{e}\right)$ term and is smaller. This is in agreement with a conjecture by Kinoshita 7 ) which suggests that constant terms from second-order vacuum polarization corrections to the fourth-order $\frac{1}{2}(g \boldsymbol{\mu}-2)$ are smaller and opposite in sign compared to the corresponding logarithmic terms. 


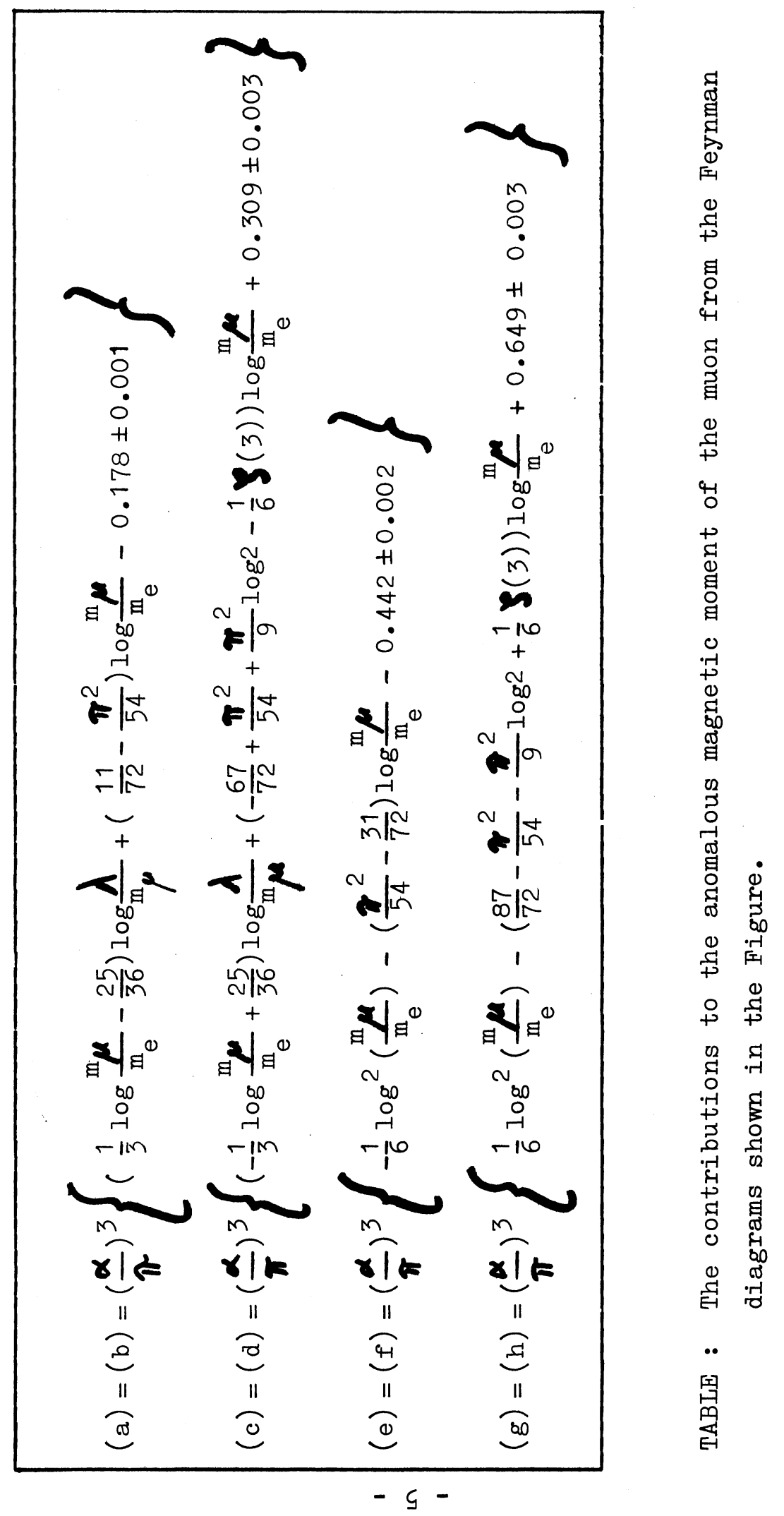


$\underline{R E E R E N L E S}$

1) The contribution from $i$ ) is known analytically. See : B.E。 Lautrup and E. de Rafael, Phys.Rev. 174, 1835 (1968).

2) The contribution from $i$ i) is known numerically. See :

JoAldins, T. Kinoshita, S.J. Brodsky and A.J. Dufner, Phys.Rev. Letters 23, 441 (1969), and SLAC preprirt, (Jan. 1970).

3) T. Kinoshita, Nuovo Cimento 51B, 140 (1967).

4) B.E. Lautrup and E. de Rafael, Nuovo Cimento 64A, 322 (1969).

5) This subroutine was originally written at CERN by G.C. Sheppey and it has been subsequently developed by A.J. Dufner and

B.E. Lautrup. A detailed description of it by these authors is under preparation. The name ISD was kindly suggested to us by M. Perrottet at a recent meeting on "Computational Methods in Theoretical Physics" held in Marseilles.

6) M. Veltman, CERN preprint (1967).

7) To Kinoshita, "The anomalous magnetic moment of the muon", in Lectures given at the Summer School of Theoretical Physics, Cargèse, 1967, edited by M. Lévy, New York (1968). 


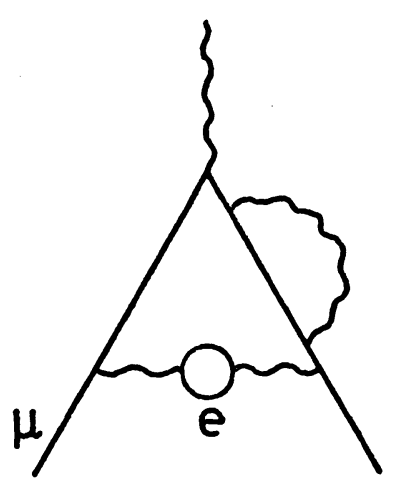

a)

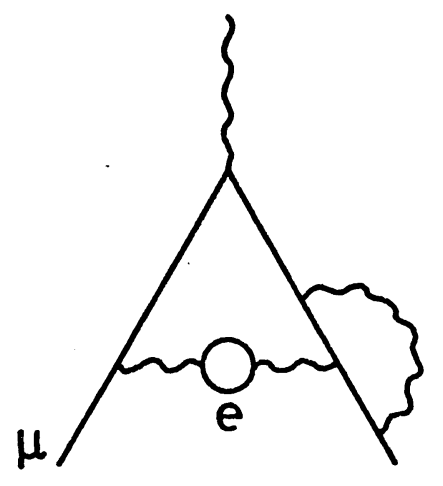

c)

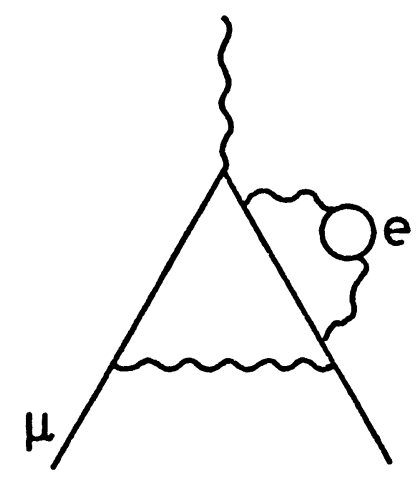

e)

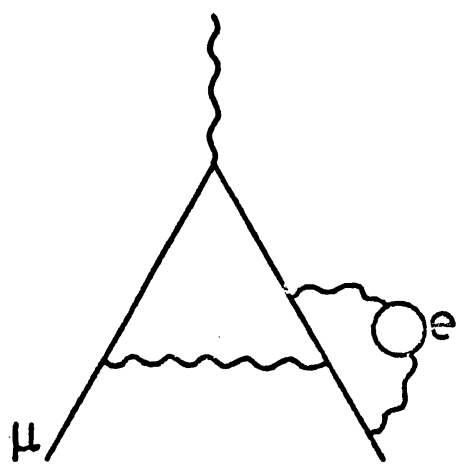

g)

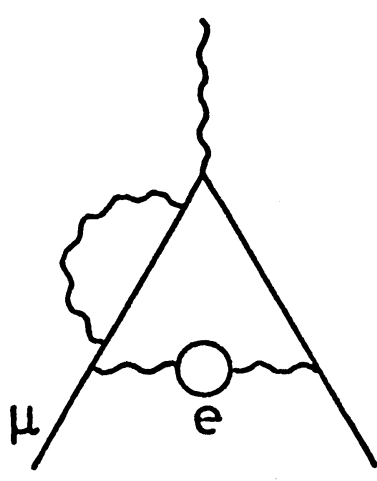

b)

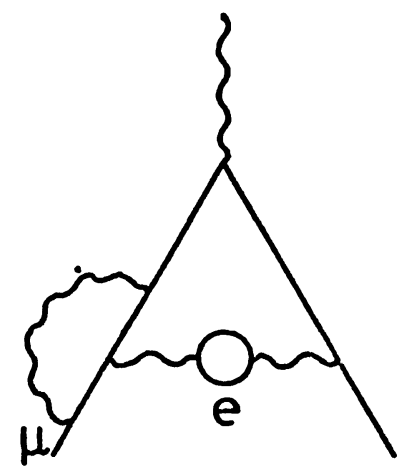

d)

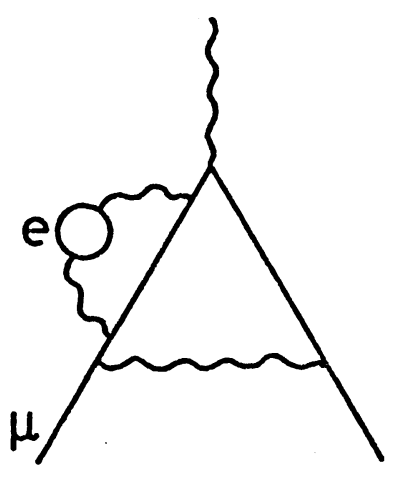

f)

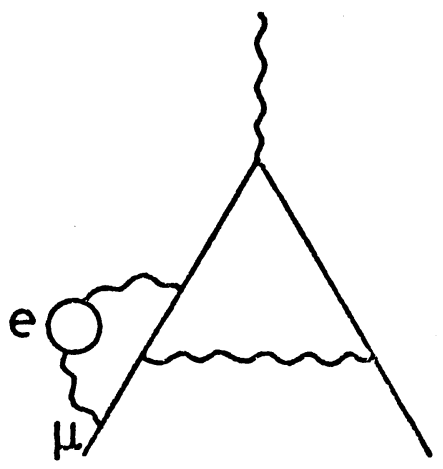

h)

FIG. 1 